\title{
Modal Adverbs Used as Hedges in Research Articles
}

\author{
Naira Martirosyan, Tatev Ghazaryan \\ Yerevan State University
}

\begin{abstract}
cademic writing is nowadays generally seen as an interaction between the writer and the reader. The main aim of the writer is to build up an interactive and constructive relationship with the readers, to persuade them of the correctness of their claims. Accordingly, the authors of research articles commonly qualify the information presented by means of items relating to vagueness, uncertainty or tentativeness. These devices are referred to as hedges.

Thus, despite a widely held view that professional scientific writing is purely objective and impersonal, hedges are abundant in science: they are a significant means for academics as they help researchers to "shield' against readers" possible rejection of their propositions.

Lakoff used the term hedge to refer to "words whose job is to make things fuzzier or less fuzzy" (Lakoff 1972:195). This definition became the starting point for several studies on this phenomenon. Later Brown \& Levinson defined hedges as "a particle, word or phrase that modifies the degree of membership of a predicate or a noun phrase in a set" (Brown \& Levinson 1987:145). They list hedging as one of various "face" preserving politeness strategies. For example, in order to avoid disagreement with the hearer the speaker can make his own opinion safely vague by using hedges such as sort of, kind of, etc.

Hyland claims that hedges are "linguistic means used to indicate either a) a lack of complete commitment to the truth value of an accompanying proposition, or b) a desire not to express that commitment categorically" (Hyland 1998:1). Thus, they mark uncertainty and help writers to present a proposition as an opinion rather than a fact.

The overview of the development of the concept clearly demonstrates that the notion of hedging has expanded by going through roughly three stages. The first stage is mainly semantic. The second stage is semantic and pragmatic, modifying the truth value of the whole proposition and the speaker's or writer's commitment or attitude to the propositional content, with its focus on the speaker-content relationship. And, finally, the third stage is mainly pragmatic and social, modifying relationships between the reader and the writer or between interlocuters, or social relationships in a wider context, with its focus on interpersonal and social relationships.

The writer's or speaker's judgments about statements and their possible effects on interlocutors is the essence of hedging, and this clearly places epistemic modality at the centre of our interest. Coates (1987:112) describes epistemic modality as concerned with “the speaker's assumptions, or assessment of possibilities, and in most cases, it indicates the speaker's confidence or lack of confidence in the truth of the proposition expressed". The epistemic system is mostly referred to as realizing a continuum of "commitment" on the part of the writer to the truth value of his or her statements, ranging from uncertain possibility to confident necessity. Its importance lies in the fact that such tentativeness
\end{abstract}


avoids personal accountability for statements, reducing the author's "degree of liability" (Huebler 1983:18), while expressing caution and facilitating open discussions. Epistemic comment is seen by many writers as being at the heart of language use and a principal means by which speakers can use language flexibly to adopt positions and express points of view. Writers cannot avoid encoding their point of view towards whatever they say, commenting on and committing to or distancing themselves from their propositions.

The concept of hedging is an area of some neglect (Crystal 1995:120) although the literature contains references to various aspects of hedging over the past 20 years. Hedges have been referred to as compromisers (James 1983), downtoners (Quirk et al 1972), weakeners (Brown \& Levinson 1987), downgraders (House \& Kasper 1981), softeners (Crystal \& Davy 1975), backgrounding terms (Low 1996) and pragmatic devices (Stubbe \& Holmes 1995). This phenomenon has received most attention in casual conversation where it is extremely common and represents a significant interpersonal and facilitative communicative resource for speakers, who use them when they wish to create an informal atmosphere, facilitate turn-taking, sustain discussion, show politeness. These units have also been treated as a form of "metadiscourse" by some applied linguists who have included them in the elements of a text.

As it has been mentioned above, hedges are abundant in science and play a critical role in academic writing. They are important to scientists because even the most assured scientific propositions have an inherently limited period of acceptance. Categorical assertions of truth in these circumstances are decidedly hazardous. What is more, the use of hedges may have provocative influence, for on the one hand such hedges as possibly, maybe, admittedly somehow signal the writer's perhaps limited knowledge, while on the other hand, hedges like certainly, undoubtedly emphasize the commitment over what would be indicated by a simple declarative in English. Hence we see that hedging is an essential element of academic writing. As Crismore \& Farnsworth (1990:15) mention: "hedging is the mark of a professional scientist, one who acknowledges the caution with which he or she does science and writes on science".

In everyday conversation hedges are commonly expressed through auxiliary verbs and by epistemic adjectives, adverbs and lexical verbs. Common conversational forms include perhaps, I guess, maybe, quite, sort of, while prosody, tag questions, hesitation markers, rhetorical questions also function as hedges when they are used to decrease the strength of an utterance (Coates \& Cameron 1988; Holmes 1995). Academic research writing also includes diverse expressions of hedging which make use of the full range of epistemic lexical resources.

In academic writing modal adverbs comprise a greater range of items than modal adjectives or nouns, from which they differ in that the former are "peripheral in clause structure" (Quirk et al 1972:421). They are not syntactically integrated as an element of the clause but can appear in a number of positions without affecting the meaning relation between the clause and the adverb. In the examples below we shall see that the modal adverb perhaps appears in all three positions without affecting the grammaticality of the sentence or changing its core meaning. In other words, the presence or absence of the modal adverb perhaps does not change the information that the speaker conveys. 
e.g.

a) Perhaps the most widely quoted ages of the universe come from the age estimates of globular cluster stars.

b) The most widely quoted ages of the universe come from the age estimates of globular cluster stars, perhaps.

c) The most widely quoted ages of the universe perhaps come from the age estimates of globular cluster stars.

(Riess et al 1998)

It is worth noting that the mobility of adverbs means that they can be selected to operate on phrasal, sentential and supra-sentential levels. On the supra-sentential level modal adverbs also perform a text-creating function, expressing various semantic relations, anaphora and cataphora included. As is known, anaphora implies retrospective connection, while cataphora indicates prospective connection. In the following examples modal adverbs illustrate anaphoric reference:

e.g.

a) But modality, as will be seen, does not relate semantically to the verb alone or primarily, but to the whole sentence. Not surprisingly, therefore, there are languages in which modality is marked elsewhere than on the verb or within a verbal complex.

(Palmer 1986)

b) With ought to/should the conditions were the carrying out of an obligation; with might they seem to be those of behaving in a sensible or proper way. But this a much stronger notion than mere permission, and it is clear that might have does not mean 'You would have acted thus if you had had permission'. Apparently then, might expresses a stronger kind of deontic modality than may - a positive suggestion rather than mere permission.

(Palmer 1986)

In these examples the modal adverbs not surprisingly and apparently refer to the preceding sentences, without which the meaning of the sentence introduced by the modal adverbs would be obscure.

Examples of cataphoric reference are:

a) In a sense, presumably, this is to be interpreted as not being direct observation; but the point is that Tajik distinguishes just two types. (Palmer 1986)

b) Intuitively, it seems reasonable to assert that an ASC II file contains 7/8 as much information as an arbitrary file of the same size, since we already know one out of every eight bits before we even look at the file.

(MacKay 2003) 
Another interesting point to be made is that modal adverbs may be modified by such words as very, too, most, quite, rather, less and may form degrees of comparison, for example:

a) Less obviously, interrogative sentences are related, not only to statements, but to relative clauses.

(Crystal 1995)

b) In notional terms all three are, in some way, concerned with the event or situation that is reported by the utterance. Tense, rather obvious$\boldsymbol{l y}$, is concerned with the time of the event, while aspect is concerned with the nature of the event.

(Palmer 1986)

c) More strictly (and significantly), this is so only if the subordinate clause is part of what is reported.

(Palmer 1986)

Research shows that modal adverbs are quite often used in combination with other modal adverbs or modal verbs:

e.g.

a) It is undoubtedly the case that most perhaps all languages have a clear way of indicating that the speaker is to be true making a statement that he believes to be true.

(Palmer 1986)

Here the writer uses the modal adverbs undoubtedly and perhaps which constitute 2 polarities, as undoubtedly expresses a strong level of modality, while perhaps - a weak level of modality.

The same can be said about the next example.

b) Although all languages probably have devices for asking questions, and although there is certainly a need to recognize the typological category of Interrogative, the ways in which questions may be expressed vary greatly.

(Palmer 1986)

On the other hand, writers may use adverbs of the same level of modality to make a greater impact on the reader.

a) The essential point here is that the absence of marking categories such as number and tense is precisely the defining characteristic of nonfinite forms, and non-finite forms typically occur in subordinate constructions. More strikingly, perhaps somewhat paradoxically, such forms are used with coordination, but not with subordination in some Papuan languages.

(Palmer 1986) 
b) Almost certainly, no synchronic explanation can be given for the use of past tense for unreality without being circular, but it may be possible to speculate, very tentatively, along the following lines, why the situation has come about historically.

(Palmer 1986)

Epistemic expressions in language, then, are likely to originate in such high-level conceptual operations as well. Hence, an investigation of them can probably not be limited to a purely linguistic description, but will obviously require a concern with the conceptual systems from which they derive.

Lyons employs the term "modally harmonic" to refer to contexts where two or more forms express the same degree of modality, such that "there is a kind of concord running through the clause, which results in the double realization of a single modality". Thus clusters overwhelmingly function to reinforce the epistemic strength of items, what Banks refers to as "fertilizing hedges".

As for the classification of hedges, it should be mentioned that there is no unanimous agreement on it, since there are no unified criteria for the classification of hedges. Prince, Fraser and Bosk (1982) classified hedging devices into two kinds: 1) approximators that express fuzziness within the propositional content, i.e. affect the truth-value of propositions; 2) shields that express fuzziness in the relationship between the propositional content and the speaker/author. Despite the seeming clearness, the taxonomy was criticized because of the obvious overlap between the categories. As Hyland has pointed out, hedging devices "do not fit into a neat scheme of discrete categories which allows one meaning to be clearly distinguished from others" (Hyland 1996:437). Moreover, meanings are frequently expressed simultaneously. The reason for this difficulty is that hedging devices are polypragmatic. The two main categories of hedges distinguished in the content of research articles by Hyland are content-oriented hedges which mitigate the relationship between propositional content and a non-linguistic mental representation of reality and reader-oriented hedges, which mostly deal with the interpersonal interaction between the reader and the writer. Now let us observe the following examples:

a) The investigation of Morgan (1998) on mathematical writing is probably the most relevant research, as it followed the same CDA framework.

(Wagner and Herbel-Eisenmann 2008)

b) Perhaps in this kind of highly standardized production environment, "thinking outside of the box" to design new and creative production processes only results in processes that are less efficient and, as a result, more variable over time.

(Hofmann and Jones 2005)

In these examples the writers make clear that they have reservations concerning whether the stated situations actually obtain.

In general, content-oriented hedges are a significant communicative resource as they enable scientific writers to both negotiate the precision of claims and convey an attitude 
to them, offering the reader a means of assessing the certainty of its truth and degree of personal commitment to it.

Summing up, it should be mentioned that the investigation is carried out on a corpus comprising research articles from three different disciplines, namely linguistics, economics and physics. We have selected 10 research articles from each discipline. The research articles we have selected had to comply with a number of requirements in order to be included in the corpus: all of them came from scientific refereed journals, their publishing span was between 1999 and 2012, the average length of each article, which has already been modified, is about 6,500 to 7,500 words. The modification includes deletion of all the material that is not the author's coherent writing such as citations, examples, or the list of references.

Our analysis of the modal adverbs used as hedges in research articles demonstrates that the frequency of their occurrence is determined by the discipline. The average number of modal adverbs used per 1.000 words is $3.5,3$ and 0.8 in linguistic, economic and physics articles respectively.

These results quite clearly indicate that modal adverbs are used slightly more frequently in linguistic articles than in economic articles and that physics articles had the lowest frequency of occurrence of hedges. Based on these findings one might speculate that, in comparison to the articles on physics, linguistic and economic articles are somewhat more flexible and less formal in their mode of expression.

The scientific paper constitutes a rhetorically competent product through which scientific knowledge is negotiated. A successful claim depends on linguistic choices which appropriately convey the writer's intention, facilitate the smooth exchange of information and create conditions for persuading readers of a point of view.

Thus, hedges are not the negative indicators of imprecision and fuzziness as many linguists think, on the contrary, they are an essential means by which speakers and writers encode a point of view.

\section{References:}

1. Brown, P. and Levinson, S. (1987) Politeness. Cambridge: Cambridge-University Press.

2. Coates, J. (1983) The Semantics of Modal Adverbs. London: Croom Helm Series.

3. Crismore, A. \& Farnsworth, R. (1990) Metadiscourse in Popular and Professional Science Discourse. Newbury Park, CA: The Writing Scholar: Studies in the Language and Conventions of Academic Discourse.

4. Hyland, K. (1998) Hedging in Scientific Research Articles. Amsterdam/Philadelphia: John Benjamins Publishing Company.

5. Hubler, A. (1985) Understatements and Hedges in English. Amsterdam/Philadelphia: Pragmatics and Beyond IV, 6.

6. Lyons, J. (1977) Semantics. Vols $1 \&$ 2. Cambridge: Cambridge University Press.

7. Lakoff, G. (1972) Hedges: A study in meaning criteria and the logic of fuzzy concepts. Chicago Linguistic Society Papers, 8 Chicago: Chicago University Press. 
8. Quirk, R.; Leech, G.; Greenbaum, S. and Svartvik, J. (1985) A Comprehensive Grammar of the English Language. London/New York: Longman.

9. Stubbe, M. \& Holmes, J. (1995) You Know, Eh and other "exasperating expressions": An Analysis of Social and Stylistic Variation in the Use of Pragmatic Devices in a Sample of New Zealand English. Elsevier Ltd: Language and Communication: 15 (1).

\section{Sources of Data:}

1. Crystal, D. (1995) The Cambridge Encyclopaedia of Language. Cambridge: CUP.

2. David, J. MacKay (2003) Information Theory, Inference and Learning Algorithms. 73. Cambridge: CUP.

3. Hoffman, D. \& Jones, L. (2005) Leadership, Collective Personality, and Performance. // Journal of Applied Psychology. Vol.90, No.3.

4. Palmer, F. (1986) Mood and Modality. Cambridge: CUP.

5. Riess et al., (1987) Observational Evidence from Supernovae for an Accelerating Universe and a Cosmological Constant. // The Astronomical Journal. Vol.116. USA.

6. Wagner, D. \& Herbel-Eisenmann, B. (2008) Just Don't: The Suppression and Invitation of Dialogue in the Mathematical Classroom. Educational Studies in Mathematics. Vol.67, No.2.

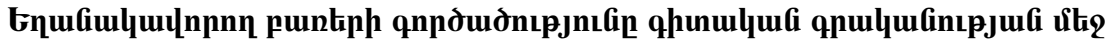

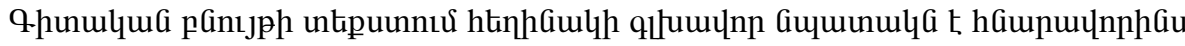

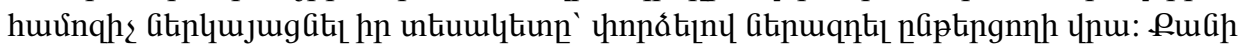

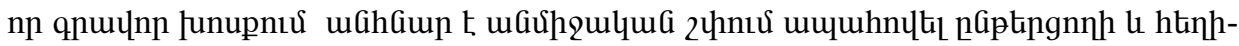

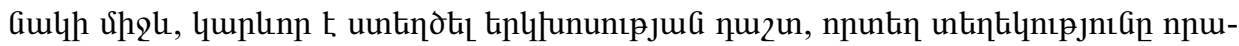

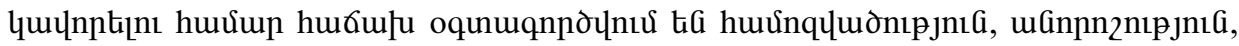

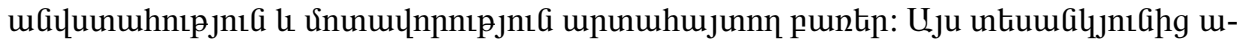

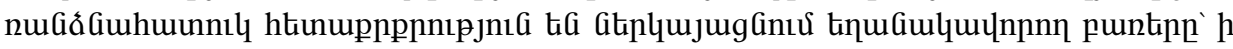

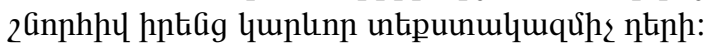

